\title{
Proceedings of Society of the British Neurological Surgeons
}

The 105th meeting of the Society was held in association with the Association of British Neurologists, in Edinburgh, 20-22 September 1984

A CRITIQUE OF THE NEUROSURGICAL MANAGEMENT OF SPINAL TRAUMA

P Harris (Edinburgh)

Mr Harris opened the meeting by discussing the problems of spinal trauma, one of the most devastating forms of injury. He referred to an incidence of up to 20 or more patients per million of the population each year, with a significant percentage of such patients having an associated injury. Policies for the management of these patients vary, but central to their care is the question of prognosis. Unfortunately some of the results of treatment in the early phase following injury had left much to be desired, and some of these poor results had been later managed by doctors who are dedicated to specialising in the multidisciplinary activities of spinal paralysis units. Mr Harris considered that, because of his training and experience in neural trauma and in other spinal disorders, the neurosurgeon had much to offer the spinal injured, in particular the necessary back-up of expert nursing staff and allied health personnel, and close relationships with colleagues in related specialities. He discussed the indications for non-operative, and for operative treatment for the traumatic spinal lesion, based on his personal experience of several years, and concluded that controlled, matched therapeutic trials were neither practical nor ethical in the human situation, and made reference to animal studies as clues to the pathophysiology and treatment.

NORMALIZATION OF ABNORMAL GLUCOSE METABOLISM OF CEREBRAL CORTEX (PET SCAN) BY CHRONIC STIMULATION OF ANTERIOR NUCLEUS OF THALMUS (FOR LIMBIC LOBE EPILEPSY) OR ANTERIOR CEREBELLUM (FOR SPASTICITY)

IS Cooper, ARM Upton, S Garnett, I Amin, G Brown, M Springman (New York and Hamilton, USA)

The authors had used positron emission tomography, employing 18F-2-fluoro-2deoxy-D-glucose. Two young adult patients underwent stimulaton of anterior lobe of the cerebellum (CCS) for treatment of spacticity of cerebral palsy. During epochs when CCS was turned off there was hyperactivity of glucose metabolism on one side of cerebral cortex and hypometabolism on the opposite side. During epochs when CCS was active there was normalisation of glucose metabolism in the entire cerbral cortex. Chronic stimulation of the anterior nucleus of the thalamus (ANT) in patients with intractable limbic system epilepsy has been carried out at Westchester County Medical Center in New York, on the basis of a hypothesis that this might interrupt seizure activity in the limbic system while prosthetically mobilising inhibitory functions of the caudate nucleus. In each of two patients studied before operations the principal abnormality was hypoactive glucose metabolism in left temporal lobe. Complete normalisation of glucose metabolism in cerebral cortex occurred only after simultaneous activation of the right and left ANT electrodes. Repeated on-off studies confirmed the cause-effect relationship. During periods of bilateral stimulation there was a statistically significant reduction in seizures as well as in EEG seizure discharges. There was a profound improvement in previously abnormal behaviour. Psychometric testing demonstrated quantitative improvement in motor function and memory. Valproic acid and dilantin blood levels rose after stimulation, requiring decreased dosages of each drug in both patients. The authors concluded that there appeared to be a close temporal correlation between the clinical effects of stimulation of either cerebellum or the anterior nucleus of the thalamus and changes in cerebral cortex glucose metabolism, as well as electro physiological, psychometric, and pharmacokinetic effects.

SUBEPENDYMOMA OF THE FOURTH VENTRICLE: A SURGICAL SERIES

R Jooma, J Bradshaw, B Brownell, M Torrens (Bristol)

Although a subependymoma of the fourth ventricle is generally considered to be an incidental post-mortem finding, the authors' clinical experience had prompted: them to draw the lesion to the attention of neurosurgeons. They presented a surgica $f_{5}^{5}$ series of 12 patients diagnosed during a 13등 year period. There were nine males, the $\frac{\bar{c}}{\bar{s}}$ average age was 56 years and the average duration of symptoms 3 years. Symptoms of bulbar dysfunction had tended to precede those of raised intracranial pressure? by many months. Five ventriculograms, $10 \overrightarrow{0}$ CT scans and one NMR scan were reviewed, in order to characterise the "typical" subependymoma. The tumous arose from the floor of the ventricle in seven cases, from the roof in two but in further two there was not an evidentoo attachment. In one patient, the origin was from the lateral recess and the lesion p sented as an angle tumour. Alteration $\$ f^{+}$ vital signs occurred during dissection of tumours attached to the floor of the ventwicle, and curtailed excision in all but one There were five post-operative deatiss each due to respiratory failure. Review the operative findings suggested that most instances the surgeon had underesti-cr mated the size of the tumour as indicate ${ }^{-}$ by radiology and as disclosed in the three cases that came to necropsy. The authors message was that the diagnosis of a subependymoma should be considered in anyo adult presenting with a fourth ventricula tumour. Even though the tumour is his tologically benign, it is usually extensive a? the time of presentation and operation is associated with hazards, in particular, a lia bility to apnoea after operation. The hoped that use of surgical tools such as the ultrasonic aspirator or laser would improve results.

MANAGEMENT OF INTRACRANIAL ABSCESS: REVIEW OF 113 CASES

ES Miller, PS Dias, D Uttley (London) Recent studies suggest that mortality of cerebral abscess may have been reduced근. The aim of this paper was to examine facf tors that might be responsible and con* versely those features still associated with of 
high mortality. The review covered the years between 1971 and 1983 and included 113 patients treated in their unit. This sug- gested an average annual incidence of $2 \cdot 9 /$ million population. The primary focus _, of infection was classified as: ENT:59 patients, thoracic: 13 patients, unknown: 30 patients, other: 11 patients. The only clinical feature significantly related to outcome was level of consciousness; 21 patients either responding only to painful stimuli or not responding, had a mortality of $60 \%$. Ninety-two patients who were obeying command had a mortality of $17 \%$. Overall the mortality was $26 \%$. The group's experience suggested that the introduction of computed tomography had made possible accurate localisation of all intracranial abscesses. Ninety-five patients were correctly diagnosed and their abscess localised before treatment, 12 were diagnosed at operation, six had abscesses either diagnosed or localised correctly only at post-mortem, of whom five had not had an operation. Sixty-three patients were treated by burr hole aspiration alone, eight had burr hole aspiration followed by excision, 32 had primary excision of the abscess, two were managed conservatively. The mortality in these groups were $20 \%$, $25 \%, 25 \%$ and $0 \%$ respectively. The only change in antimicrobial therapy had been the introduction of metronidazole. The authors re-emphasised that future attempts to improve in management must be directed towards earlier recognition and speedier referral of cases and prompt . neurosurgical management.

FIFTY YEARS EXPERIENCE WITH CHORDOMAS IN SOUTH-EAST SCOTLAND

P O'Neill, BA Bell, I Jacobson, JD Miller (Edinburgh \& Dundee)

Chordomas are relatively rare malignant neoplasms, the mean annual incidence in one series being 0.3 males and 0.18 females per one million population. This 'limits an individual clinician's experience with the tumour. The authors therefore reported the clinical presentation and management of 34 patients with a histologically proven chordoma, who had been treated in the neurosurgical departments in Edinburgh and Dundee, over the past 50 -years. The tumours involved three main areas of the neuraxis: intracanial $(35 \%)$, sacro-coccygeal (53\%) with only four $(12 \%)$ in the rest of the vertebral column. With an intracranial tumour, cranial nerve palsies were almost invariable as presenting signs. In contrast, the presentation of sacrococcygeal tumours seemed to be related more to the mass effect of the lesion than to its capacity to involve adjacent neural structures, so that back pain, bowel disturbance and a palpable mass were each relatively common. The mean survival in patients with an intracranial chordoma was 7.7 years, with sacrococcygeal chordomas was $7 \cdot 2$ years. There were too few vertebral tumours to provide a reliable estimate of mean survival time. The Edinburgh and Dundee workers experience led them to recommend aggressive surgery and radiotherapy as the optimum treatment. The combination of hyperthermia and chemotherapy had shown some promise in a few cases but had not been tested in a sufficient number of patients. Definitive studies would require a multicentre trial and extremely long follow-up.

CAROTID LIGATION - WHAT HAPPENS IN THE LONG TERM?

AN Jha, P Butler, RA Fawcitt, RH Lye (Manchester)

Common carotid ligation is occasionally used in the treatment of giant or inaccessible intracranial aneurysms. There have been few long-term studies of patients who have undergone this procedure. The authors reviewed a series of 115 patients who, over the period 1954-1984 underwent common carotid ligation. Seventythree of the patients presented with a subarachnoid haemorrhage and 42 patients had mass effects from an unruptured aneurysm. Average age at ligation was $\mathbf{5 0}$ years (22-77 years) and of a total of 141 aneurysms demonstrated, the distribution was: posterior communicating artery 65 ; other internal carotid artery sites 45; anterior communicating artery 20 ; middle cerebral artery 11 . Multiple aneurysms were present in 16 patients. Case histories were reviewed and patients recalled. Thirty-nine patients were lost to follow-up after a variable period (mean 3.75 years) Of the remaining 76 patients, 26 had died. Nineteen of the 26 deaths were 6 months or more after ligation and 13 of these were caused by proven or suspected recurrent haemorrhage. Check angiograms were obtained from 55 patients after a mean interval of 8.4 years following carotid ligation (42 patients underwent digital intravenous arteriograms; 13 had conventional angiography). Of 67 aneurysms present before ligation, 40 were not seen, 11 were smaller, 15 were unchanged and one aneurysm had enlarged. New aneurysms were noted in four patients. Annual mortality for those patients traced was $1.5 \%$ and the projected annual mortality (lost to follow-up, presumed dead) was $4.3 \%$. Common carotid ligation may offer early protection from fatal recurrent haemorrhage and it appears to reduce the size of many aneurysms. However, the authors concluded that the procedure does not appear to reduce significantly the long term prognosis for fatal recurrent haemorrhage compared with that of the natural history of anterior circulation aneurysms.

FURTHER EXPERIENCE IN STEREOTACTIC PONTINE TRACTOTOMY

ER Hitchcock, MC Kim, MG Sotelo (Birmingham)

The value of cordotomy for the relief of intractable pain is well established but it is not always possible to limit the destruction to the spinothalamic tract and avoid injury to associated fibres. Damage to the descending respiratory pathway is particularly dangerous and cervical cordotomy often fails to relieve pain at high levels. Stereotactic radiofrequency pontine spinothalamic tractotomy has the advantage that the separation of autonomic and pain pathways at pontine level permits high analgesic levels avoiding any accompanying disorders of micturition or respiration The authors reported that they had found stereotactic pontine tractotomy most suitable for recurrent intractable pain, needing high level analgesia and in patients with poor respiratory function. The procedure was less complex and difficult than might appear but precise stereotactic instrumentation was essential. Experience with selected patients was described, and illustrated that reliable pain relief of long duration without gross complications and with well sustained analgesia could be achieved.

CRANIAL DURAL ARTERIO-VENOURS FISTULAE

D Gentleman, E Teasdale (Glasgow)

Cranial dural arteriovenous fistulae were first described in 1931 , but were infrequently diagnosed before the introduction of superselective catheter angiography in the 1970s. In order to illustrate the clinical features, management, and outcome of treatment, the authors reported five male and three female patients; the mean age was 54 (range 30-69). Four presented with sudden headache, and one each with grand mal seizures, sudden loud bruit, unilateral 
proptosis and ophthalmoplegia, and finally drowsiness following head injury. When admitted to hospital, three patients had altered conscious level, three had focal neurological deficits and three had a cranial bruit. Five were considered to have had an intracranial haemorrhage. Cerebral CT scanning, showed an intracranial haematoma in four cases and a dilated vessel in a fifth. In each patient, catheter angiography of the carotid and/or vertebral circulation demonstrated the dural fistula, and in seven cases the lesion was embolised with Lyodura after superselective catheterisation. The remaining patient's fistula was supplied chiefly by the internal carotid artery. Sixteen angiographic procedures were carried out in these seven patients. Three became asymptomatic after one embolisation, and another after two. The earliest patient required five embolisations to abolish her symptoms. The sixth patient had recurrent tinnitus after each of four procedures, and in the seventh patient only one out of two major feeding vessels could be embolised; this patient is awaiting further treatment. Complications of embolisation were: one patient had a transient hemiparesis, and two had facial pain for several weeks. At follow-up between six and 35 months later, all patients were alive, and none had suffered a recurrent haemorrhage. One had continuing fits, and another had persistent tinnitus. Their experience led the authors to conclude that dural fistulae were often complex and in sites that cautioned against surgical removal; they considered that therapeutic embolisation, via superselective catheter angiography, offered an alternative method of treatment that was safe and usually effective.

THE CLINICAL SPECTRUM OF HEAD INJURY JD Miller, P Jones (Edinburgh)

Current interest in the management of head injury focuses on policies for admission and skull radiography, and for transfer to neurosurgical units, and CT scanning, also the prevalence of systemic insults and avoidable intracranial complications. Because of selective admission policies, experience in most neurosurgical units in the UK is biased heavily toward the most severely head injured. The practice in Edinburgh, where all head injured patients admitted to the Royal Infirmary are under the care of the regional Department of Surgical Neurology, had provided the authors with an opportunity to service the full clinical range of head injury. They compared 1919 cases of head injury admitted in 1981 with 1492 cases admitted in 1982 , after their admission policy had been changed, and 1179 cases admitted in 1983, during most of which time the revised seat belt legislation was in force. Patients were subdivided into mild, moderate and severe categories according to the admission score on the Glasgow Coma Scale. Of the 1919 patients admitted in $19811616(84 \%)$ were mild ( 7 deaths), 210 (11\%) moderate (6 deaths) and 93 ( $5 \%$ ) severe (42 deaths). The change in admission policy, permitting discharge from $A \& E$ of certain patients with a history of loss of consciousness, was followed by a marked fall in head injury admissions. This was confined to mild cases, and the number of moderate/severe cases and the mortality were not significantly affected. Similarly, although the enactment of new seat belt legislation was followed by a reduction in the numbers and severity of injured in drivers and front seat passengers, the total numbers of moderate and serious head injury cases and the total head injury mortality did not change. The authors emphasised that, although few in proportion to overall numbers, there were many patients with apparently minor injuries who did have other major problems and that the proper care of these called for a major commitment in time and resources.

\section{THE PROVENANCE OF EXTRADURAL} HAEMATOMA

D Sandeman, B Cummins (Bristol)

The publication of guidelines, devised by a group of neurosurgeons, for the management of head injuries had prompted a review of 100 extradural haematoma (EDH) who had presented to the authors' unit in the last ten years. Fifty seven were adults and 36 children. Thirty-seven patients presented to the referring Accident Centre with the signs of an extradural already developing; 55 patients developed signs while under observation. All the children and 42 of the adults developed the signs of EDH within 48 hours of the injury. In the remaining adults the diagnosis was delayed until after 48 hours. Only five of these developed clinical signs; the other 10 had a CT scan because of persisting symptoms, without neurological signs. Only two patients showed the classical lucid interval between periods of unconsciousness, $93 \%$ of adults had a skull fracture but $33 \%$ of children did not. The published criteria for admission and skull radiography were applied retrospectively to each patient.
Eighty-nine of the patients would have been admitted, 64 because of confusion or depression of the level of consciousness on initial assessment, seven because of neurological symptoms or signs alone and 19 because of skull fracture alone. Of the latter, 10 would have had radiography because of a history of unconsciousness or amnesia, three because of a suspected CSF leak, but in five the only indication for radiography would have been the presence of a scalp haematoma or laceration. Four patients would not have been admitted. In two a skull fracture was missed on radiography. The other two cases were children who did not have skull fractures. (Again the only indication for radiography of these children was the presence of a scalp contusion). In one of these four patients the clinical diagnosis of EDH was made three days after injury, but the other three developed their signs less than 36 hours after injury. The authors emphasised two points: (1) That the diagnosis of a skull fracture was crucial in the assessment of alert adult patients and that often the only clue to a fracture was a scalp haematoma or laceration. (2) That there were difficulties in the assessment of children but that most chits os dren should develop signs of the the of extradural within a 48 hour period of observation. They concluded that a용 patients with a scalp laceration or contw sion should have skull radiographs and thaे great care must be taken with the asses용 ment of head injured children.

CORRELATION OF TARGET SITE WITH HISTOLOGY AND CELL CULTURE IN CT-DIRECTED STEREOTACTIC BIOPSY

M Powell, J Olney, J Darling, D Thomas (London)

Initial clinical experience of the use of the Brown Roberts Wells frame for CTdirected stereotactic brain biopsy had been reported previously to the Society. In this second series, the group's aims had been to study histological variation in relation to CT biopsy site and also to correlate this ? with cell culture. They had studied 38 . cases: 26 gliomas, 3 secondary carcinomas, 5 miscellaneous "tumours" (Sarcoid, Ger- $ᄋ$ minoma, Pinealoma, Craniopharyngioma, and Primary brain lymphoma) and four 0 cases where identifiable tumour was not obtained. They considered that CT biopsy $\frac{D}{2}$ had remained relatively safe with no deaths but there had been four deteriorations, two $N$ temporary, attributable to the procedure. They had taken biopsy specimens from at $N$ 
least two sites within the tumour: the centre and the enhancing edge. In 15 of the 34 tumours $(44.1 \%)$ there were clear histological differences between tumour centre and its edge as defined by CT. Indeed in $10(29.4 \%)$ cases the edge was histologically tumour free. In the 26 gliomas, $13(50 \%)$ cases showed a histological difference and $8(30.8 \%)$ were tumour free at the edge. They concluded, tentatively, that the brain beyond the apparent enhancing boundary on CT may be free of tumour even in a malignant glioma. The results of cell culture neither confirmed nor denied their hypothesis. Of 36 patient samples $10,(28 \%)$ failed to grow; seven of these were from tumour types which rarely grow in culture (eg craniopharyngioma). In one case where an equivocal histological report was received a tumour was grown, and in another, the histological tumour free edge also grew cells. Both of these would be explained by slight differences in single target sites.

CLINICAL ASSESSMENT OF PATIENTS WITH SUBARACHNOID HAEMORRHAGE

G Teasdale, K Lindsay, S Dharker, G Mill Existing scales for grading patients with a recent subarachnoid haemorrhage have a high inter-observer variability. The World Federation of Neurosurgical societies has established a committee with the task of searching for a clearly defined, practical way of assessing patients. One proposal being studied is that an assessment of the

- Glasgow Coma Scale should be combined with other clinical features of intracranial haemorrhage (Teasdale, Lindsay and Knill-Jones, Neurosurgery 1983). To examine if this can be a useful index the authors analysed, in a retrospective study, the incidence of different degrees of impaired consciousness, of features of men-

-ingism such as headache and neck stiffness, and of focal deficits. They had studied in 141 patients the frequency of different combinations and how these correlated

- with outcome and with previous scales. In the light of the results a simple five point scale was proposed.

The authors reported that when there were differences in scores on previous scales and the new method, the latter usually provided the more appropriate index of prognosis and also had a higher inter-observer consistency. They recommended it as a simple and effective method for assessing patients with subarachnoid haemorrhage.

SEQUENTIAL CEREBRAL BLOOD FLOWS FOLLOWING SUBARACHNOID HAEMORRHAGE IS IT AN AID TO MANAGEMENT?

EW Mee, DE Dorrance, LZ Barsoum, DG Hunter, G Neil-Dwyer (London)

The object of this prospective study was to ascertain if daily serial measurement of cerebral blood flows (CBF) had a role in the clinical management of patients who had suffered a subarachnoid haemorrhage (SAH). The 23 patients, from a sequential series of 50 admitted to the authors unit, were those who proceeded to surgery for clipping of an intracranial aneurysm and on whom daily pre-, per- and post-operative measurements had been made, using the 133 Xenon rebreathing technique. The patients clinical grade and blood pressure were also recorded daily. The presence or absence of blood on the CT scan and cerebral angiographic findings were also noted. The CBFs were adjusted for age and sex and were expressed in terms of the number of standard deviations by which they differed from the expected. The study was divided into three phases: (a) preoperative, (b) peroperative and (c) postoperative. Three outcomes recognised: (a) seven patients who had no postoperative complications and made a full recovery, (b) 12 patients who had postoperative complications but who made a full recovery and (c) four patients who had major postoperative complications and have major neurological deficits more than three months postoperatively or who have died $(n=1)$. The results

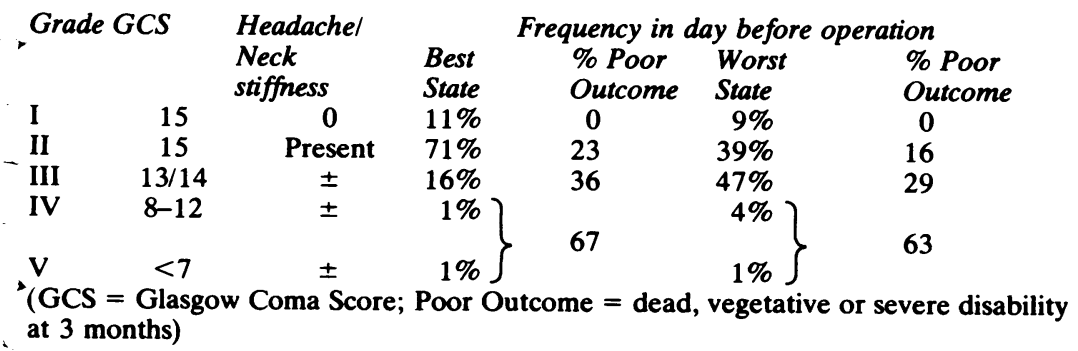

showed the following general patterns. In patients whose CBFs were rising at the time of surgery there were fewer postoperative complications and all had a good outcome. By contrast, if the preoperative flows were falling at the time of surgery there were more frequent and severe postoperative complications and the final outcome tended to be poorer. These observations were independent of the patients preoperative clinical condition. Peroperatively falling $\mathrm{CBF}$ measurements were indicative of more postoperative complications and a poorer outcome whilst rising flows suggested fewer postoperative complications and a better outcome. The authors suggested their preoperative $\mathrm{CBF}$ measurements might be useful in clinical management, a negative slope being an indication of a stormy postoperative course and/or poor outcome, whilst a positive slope is suggestive of fewer postoperative problems and a good recovery.

DIPYRIDAMOLE IN POST-OPERATIVE VASOSPASM

MDM Shaw, PM Foy, JD Pickard, M Conway, J Spillane, DW Chadwick (Liverpool and Southampton)

Cerebral ischaemia remains a potent cause $\overparen{(D)}$ of death and disability after surgery for aneurysmal subarachnoid haemorrhage. Vasospasm has been considered to be the $?$ cause but some recent evidence suggests that the basic underlying problem may be proliferative vasculopathy. The authors noted that, although the role of the platelets in this event is not clear, release of platelet factors into the vessel wall could result in medial hypertrophy, and hence to vessel narrowing. Furthermore, the disaggregation that occurs could during platelet thrombus formation lead to the release of small platelet emboli. Dipyridamole can reduce new platelet aggregation and reverse platelets adherence at the site of a vascular injury. These observations had led to a collaborative randomised trial to discover if dipyridamole reduced the frequency of ischaemic complications following surgery for aneurysmal subarachnoid haemorrhage. Six hundred and seventyseven patients were randomised, of whom 348 underwent surgery. There was not a significant difference between the patients randomised to dipyridamole as compared with those on placebo, in respect of their pre-operative clinical condition, grading (Hunt and Hess), and peroperative findings; however more patients on placebo had subarachnoid blood whilst 
more in the dipyridamole group had tight, stiff brains. The results showed that the outcome of the dipyridamole and placebo groups did not differ significantly with respect to mortality or morbidity (Glasgow Outcome Scale). No untoward haemostatic problems were found in the dipyridamole group and in particular there was no evidence of an increased risk of rehaemorrhage pre-operatively. The results therefore did not support the use of dipyridamole in this dosage, but did not exclude a role of platelets in vascular events after subarachnoid haemorrhage.

EXTRAOPERATIVE CORTICAL MAPPING IN EPILEPTIC PATIENTS USING CHRONICALLY IMPLANTED SUBDURAL ELECTRODES

IA Awad, JF Hahn, R Lesser, H Lueders (Cleveland, Ohio, USA)

The surgical treatment of seizures requires maximum resection of epileptogenic cortex while sparing functionally vital tissue. In practice, recognition of the optimum line of resection is difficult. Forty-eight patients underwent a craniotomy during which subdural electrodes were implanted. The patients were then maintained on intravenous antibiotics for a mean of 13 days, during which they underwent intensive monitoring. Using the implanted electrodes, the motor and speech areas were mapped by cortical stimulation: the sensory and auditory areas were mapped by evoked potential recording: and, precise localisation of epileptogenic cortex was accomplished by direct extraoperative electrocorticography. Patients then underwent a second craniotomy, the electrodes were removed, and the seizure focus was excised. The authors found that there was marked variability of cortical locatisation in individual patients. Also, the seizure focus was not always limited to temporal lobe, and occasionally involved frontal, parietal, or occipital areas. The standard "temporal lobectomy" using classical cortical landmarks would have failed to resect the mapped seizure focus in of 12 of 48 patients and would have resected cortex defined as functional in 18 patients. The authors considered that the advantages of the method included the feasibility of extensive and unhastened monitoring outside the operating theatre, the avoidance of craniotomy under local anaesthesia, and provision of a rational and extensive resection strategy for each individual patient. These outweighed the disadvantages, which included the risks of infection (two patients), and electrode intolerance, which necessitated early removal of electrodes in two patients.

DEMENTIA DUE TO MENINGIOMA - OUTCOME AFTER SURGICAL REMOVAL

CP Chee, AS David, SL Galbraith, RA Gillham (Glasgow)

Dementia is a common presentation of patients with an intracranial meningioma. The condition has usually been present for a long time, and it is customary to be cautious about the prospects of improvement after operation. The authors drew attention to the lack of a systematic study of the outcome of such cases and therefore had studied the effects of operation in patients with dementia was due to meningioma. The study was retrospective, and dementia defined as a progressive disintegration of intellect, of memory and of the powers of abstract thought. Between June 1981 and October 1983, 38 patients were diagnosed to have an intracranial meningioma, 10 $(26 \%)$ of these had presented with dementia. The family of a 79 year old man did not wish him to have an operation, leaving nine cases for study. One patient died from a pulmonary embolus two weeks after operation. Another who had a middle third parasagittal meningioma remained akinetic and mute post operatively and died six months later. Of the remaining seven patients, five were described as normal at follow-up six months later. Formal psychometric assessment in four of these patients confirmed the intellectual improvement. One other had improved but remained disinhibited and one did not improve. Incontinence was cured in three of four patients. The authors concluded that their findings showed that after removal of a meningioma improvement from dementia was usual, but not uniform. Although two patients died without recovering, at least half recovered, apparently fully.

FORTY TWO CASES OF INTRACRANIAL OLIGODENDROGLIOMA IN CAMBRIDGE IN 1961-1984

IMS Wilkinson, AE Holmes (Cambridge) The authors had collaborated to review presenting features, management and outcome of 41 patients with an oligodendroglioma. There was an equal sex distribution and most patients presented between the ages of 30 and 50 . Epilepsy was the most common first symptom, sometimes of long duration ( 19 out of 42 cases). Half gave a history of less than twelve months duration. Patients with a short history often had raised intracranial pressure, or an oncoming neurological deficit. Twenty tumours were frontal in position, nine temporal or fronto-temporal, and 13 more posteriorly situated. Half the tumours were calcified at the time of diagnosis. Mitotic activity was observed in most tumours (36 out of 42 cases). Operation was performed in each case to establish the diagnosis. Eight patients had a needle biopsy through a burrhole, three had an open biopsy at craniotomy, 17 had a subtotal removal and 14 had a macroscopic complete removal. Five cases had further operations, 31 patients had radiotherapy after initial surgery and five at a later date. The outcome was not as favourable as anticipated; eleven patients died within three months of operation and only ten patients survived five years. No specific clinical, operative or histological factors appear to predict the length of survival.

STUDIES ON CEREBRAL FUNCTION AN BLOOD-BRAIN BARRIER PERMEABILITY PATIENTS WITH HYDROCEPHALUS ANZ BENIGN INTRACRANIAL HYPERTENSION C USING POSITRON EMISSION TOMOGRAPHY $\bigcirc$ DJ Brooks, RP Beaney, KL Leender M Powell, A Crockard, DGT Thoma șidsa J Marshall, T Jones (London)

The authors had used positron emission tomography to study cerebral oxygen utilisation and blood flow in patients with acute obstructive hydrocephalus, with normal pressure hydrocephalus or hydrocephalus secondary to congential lesions, or with benign intracranial hypertension. Patients with hydrocephalus due to all causes showed a reduction in cortical oxygen utilisation and blood flow compared to normal controls, but those patients whose symptoms had been present for four months or less had a raised oxygen extraction, considered to be an indication of a potentially reversible situation. Both acute and chronic groups of hydrocephalus patients were restudied after cerebral decompression. The acute group showed a significant increase in cortical blood flow and a fall in cortical oxygen extraction following surgery, whereas little change was found in the cerebral metabolism in the chronic group. Patients with benign intercranial hypertension and CSF pressures of up to $58 \mathrm{~cm}$ of water did not show 
significant changes in cerebral oxygen utilisation, blood flow or cerebral blood volume. Blood-brain-barrier permeability was assessed using $82 \mathrm{Rb}+$, a $\mathrm{K}+$ analogue, but

- was not abnormal either in patients with benign intracranial hypertension or in -those acute obstructive hydrocephalus. Although clinical correlations were not reported, the authors suggested that a raised oxygen extraction by cortical tissue 'might indicate potentially reversible cerebral dysfunction in hydrocephalus. Nevertheless only those patients with a short history of symptoms were likely on - metabolic grounds to respond to cerebral decompression.

RECIRCULATION FOLLOWING TEMPORARY CEREBRAL ISCHAEMIA

NV Todd, P Picozzi, HA Crockard, R Ross-Russell (London)

-Transient cerebral ischaemia is seen by neurologists as a result of spontaneous emboli from the cortid artery and by neurosurgeons as a result of temporary

- vascular clipping during surgery for aneurysm and AVM. This study examined the

- effect of increasing duration of ischaemia on the level of blood flow during recircula-tion. Global forebrain ischaemia was produced in anaesthetised rats (Pulsinelli 1979) by permanent occlusion of the vertebral artery followed by temporary carotid clamping, CBF was measured regionally by hydrogen clearance. Three groups of rats swere made ischaemic for 15,30 , or 60 minutes (Groups A, B, C). During carotid -occlusion CBF fell in all rats, but there were differences in the extent of reflow between the three groups. On recirculation there was a pronounced but short lasting Group A, but most prolonged in group $\mathrm{C}$. This was followed by a prolonged reduction in CBF (hypoperfusion) with flow - reduced most in Group $A$ and least in Group C. These differences were found at $\mathbf{1}, 2$, and 3 hours of recirculation. hyperaemia which was most marked in

The authors concluded that even short periods of temporary vasular clipping could be followed by an initial hyperaemia and finally by hypoperfusion with a severe reduction in flow ( $60 \%$ control values) lasting for many hours after recirculation.

CHRONIC SUBDURAL HAEMATOMA IN THE ELDERLY - SHOULD WE OPERATE?

RDE Battersby (Sheffield)

Management of chronic subdural haematoma in the elderly is subject to controversy: are the presenting symptoms due to the lesion or to longstanding central nervous system disorder? is surgical treatment worthwhile? are more patients being referred? To answer these questions the author had studied 72 patients aged between 60 and 90 years treated between 1957 and 1983. Thirty-eight of the patients were in the seventh decade, 19 in the eighth and 15 in the ninth decade. A four fold increase in referrals has occurred since 1975, with average age and porportion of females rising significantly. Follow up was for 2 years, or until full independence was achieved. Older patients more often had confusion than raised intracranial pressure as a presenting symptom. Focal symptoms were equally distributed. A history of an injury was obtained in $67 \%$ and was more likely with increasing age. Age did not influence duration of symptoms or site of lesion. In the seventh and eighth decades full recovery occurred in $89 \%$, with a surgical mortality of $3.5 \%$; in the ninth decade full recovery occurred in only $27 \%$ and mortality was $47 \%$. Six patients had additional longstanding CNS disorders (eg confusion); five of these were in their eighties and none benefited from surgery. Outcome overall depended on age, with older patients faring worst, but not on duration of presenting symptoms, nor on acute confusion or coma on admission. Patients over 80 often had symptoms attributable to both longstanding CNS disorder and a coinci-

\begin{tabular}{lccc}
$\begin{array}{l}\text { Group } \\
\text { Duration of Ischaemia (minutes) }\end{array}$ & $A$ & $B$ & $C$ \\
\hline Pre-occlusion blood flow & 15 & 30 & 60 \\
Ischaemic blood flow & $80 \pm 5$ & $93 \pm 9$ & $96 \pm$ \\
Peak hyperaemia & $256 \pm 13$ & $186 \pm 20$ & $167 \pm 15$ \\
Duration of hyperaemia (min) & $16.9 \pm 0 \cdot 5$ & $21.9 \pm 1 \cdot 3 \dagger$ & $62 \cdot 1 \pm 7$ \\
rCBF at 60 mins reperfusion & $44 \pm 6 \cdot 5^{*}$ & $48 \pm 3 \cdot 5$ & $82 \pm 12.9 \dagger$
\end{tabular}

$* p<0.01 \quad$ tp $<0.001$ dental subdural collection; below this age symptoms tended to be more clearly defined. It was concluded that surgery should be recommended in all patients below 80 years and in all patients with raised intracranial pressure, but the author cautioned that very elderly patients, especially those with longstanding CNS disorder, had a poor prospect of useful recovery.

A RETROSPECTIVE COMPARATIVE STUDY OF MEDICAL VERSUS SURGICAL MANAGEMENT OF GLIOMAS

SJ Roe, GMcD Towns, DW Chadwick, PM Foy, MDM Shaw, IR Williams (Liverpool) The doubts about the value of surgery for malignant gliomas had led to a great reluctance on the part of the medical neurologists in Liverpool to refer patients for a biopsy. The practices of two neurologists were compared with those of two neurosurgeons, all of whom considered themselves to be conservative in their management of gliomas. They reviewed retrospectively all CT scan reports for such cases admitted under the care of the four consultants over the 5 year period 1978 to 1982 , and also the case notes of all patients whose CT scan report raised any possibility of the diagnosis of a brain tumour were reviewed retrospectively. Those patients in whom the diagnosis of a primary malignant brain tumour was made on the basis of clinical and CT findings were included in the study. Patients with proven primary malignancies elsewhere and multiple intracerebral lesions were excluded. Two hundred and eight patients were identified but to July 1984 complete follow-up was achieved in 167 patients and these formed the basis of this preliminary report. Patients were grouped according to whether initial referral had been to a neurosurgeon (MDMS, PMF) or a neurologist (DWC, IRW). Comparison revealed no differences with respect to age, sex, clinical features including Glasgow Coma Scale rating at the time of referral, CT scan appearances, outcome on leaving hospital according to the Glasgow Outcome Scale and time of death or current follow-up. Neurologists and neurosurgeons, having been referred similar patients, differed in the number of patients selected for burrhole biopsy. In consequence histological confirmation of the diagnosis was made less often in the neurological patients, but analysis of the pathological findings suggested that only the astrocytomas in the neurologically referred 
patients were not being biopsied. Young patients and those with cystic lesions were more likely to be biopsed no matter to whom they were referred. Burrhole biopsy was associated with an increased early mortality and morbidity but there were a few long term survivors amongst those biopsied. Referral to a neurologist or neurosurgeon did not influence whether or not a patient underwent open biopsy with or without an internal decompression. The authors concluded that there are some patients in whom there is little reasonable doubt as to the diagnosis on clinical and CT grounds and in whom histological confirmation is not necessary for subse- quent management.

The Norman Dott Memorial Lecture was $\mathbb{D}$ delivered during the meeting by Dr Paul Bucy, Chicago. The title of his address was $₫$ "Scotland - The Birthplace of Surgical Neurology". 